\title{
Directed Forgetting and Directed Remembering in Visual Working Memory
}

\author{
Melonie Williams and Geoffrey F. Woodman \\ Vanderbilt University, Vanderbilt Vision Research Center, Center for Integrative and Cognitive \\ Neuroscience
}

\section{Abstract}

A defining characteristic of visual working memory is its limited capacity. This means that it is crucial to maintain only the most relevant information in visual working memory. However, empirical research is mixed as to whether it is possible to selectively maintain a subset of the information previously encoded into visual working memory. Here we examined the ability of subjects to use cues to either forget or remember a subset of the information already stored in visual working memory. In Experiment 1, participants were cued to either forget or remember one of two groups of colored squares during a change-detection task. We found that both types of cues aided performance in the visual working memory task, but that observers benefited more from a cue to remember than a cue to forget a subset of the objects. In Experiment 2, we show that the previous findings, which indicated that directed-forgetting cues are ineffective, were likely due to the presence of invalid cues that appear to cause observers to disregard such cues as unreliable. In Experiment 3, we recorded event-related potentials (ERPs) and show that an electrophysiological index of focused maintenance is elicited by cues that indicate which subset of information in visual working memory needs to be remembered, ruling out alternative explanations of the behavioral effects of retention-interval cues. The present findings demonstrate that observers can focus maintenance mechanisms on specific objects in visual working memory based on cues indicating future task relevance.

\footnotetext{
Our ability to temporarily store information in memory is a fundamental limit of our cognitive system. Studies using change-detection tasks have shown that the average capacity of visual working memory is approximately 3 to 4 object representations (Irwin \& Andrews, 1996; Luck \& Vogel, 1997; Vogel, Woodman, \& Luck, 2001). Because of this highly constrained capacity, it is important that we hold representations of only the most relevant information in visual working memory and remain sensitive to cues from the environment regarding future relevance. A body of research suggests that we benefit from such cues because they allow us to focus maintenance processes (perhaps via the deployment of visual attention) on the most relevant objects represented in memory (Averbach \& Coriel, 1961; Griffin \& Nobre, 2003; Landman, Spekreijse, \& Lamme, 2004; Makovski \& Jiang, 2007; Makovski, Sussman, \& Jiang, 2008; Matsukura, Luck, \& Vecera, 2007; Schmidt, Vogel, Woodman, \& Luck, 2002; Sperling, 1960; Woodman, Vecera, \& Luck, 2003). However, some research using cues that direct participants to forget a subset of the information stored in visual working memory has suggested that there is no active rehearsal of the information being maintained (Bays \& Husain, 2008; Proctor, 1983; Shaffer \& Shiffrin, 1972; Washburn $\&$ Astur, 1998). The goal of the present study was to determine whether control can be exerted over the maintenance of representations already held in visual working memory.
}

Correspondence from the editor/publisher should be addressed to: Geoffrey F. Woodman, Department of Psychology, Vanderbilt University, PMB 407817, 2301 Vanderbilt Place, Nashville, TN 37240-7817, 615-322-0089 (telephone), 615-343-8449 (fax), geoffrey.f.woodman@vanderbilt.edu. 
Determining whether we can control which items receive the benefit of maintenance after encoding a larger set of information into visual working memory is of critical theoretical importance. Theories of visual-spatial working memory have proposed that object representations are actively rehearsed by just such a limited-capacity spatial mechanism (i.e., the inner scribe of Baddeley \& Logie, 1999; Logie, 1995). The general idea is that this spatial mechanism traces over the object representations to refresh their features, analogous to proposals about the rehearsal of verbal information in the phonological loop (Baddeley, 1986). Several prominent models of cognition propose that the contents of working memory are simply the activated representations in long-term memory (Lovett, Reder, \& Lebiere, 1999). Such models are particularly invested in the notion that we can voluntarily maintain certain pieces of information in working memory and not others. For example, the embedded-process model of working memory proposed by Cowan $(1997 ; 1999)$ also proposes that we can voluntarily enhance or inhibit representations stored in working memory. This model is designed such that the focus of attention helps to maintain the activation of representations in memory. In addition, the limited-capacity focus of attention is controlled by executive mechanisms that can voluntarily redeploy attention to particularly relevant representations. The embedded-process model explicitly proposes that attention not only can be directed to certain representations to maintain their heightened activations, but also directed away from particular items in memory, allowing it to account for existing evidence (Zacks \& Hasher, 1994). Thus, a fundamental prediction of theories of working memory is that we should be able to act upon cues to maintain certain information and evidence to the contrary would necessitate the revision of many of these theories.

A body of empirical evidence does support the idea that we actively rehearse information in visual working memory by voluntarily enhancing what we are trying to remember. First, consider how spatial locations are maintained in visual working memory. A series of studies by Awh and colleagues (Awh, Anllo-Vento, \& Hillyard, 2000; Awh \& Jonides, 2001; Awh, Jonides, \& Reuter-Lorenz, 1998) has shown that visual-spatial attention can be used to aid the maintenance of spatial locations representations in visual working memory (i.e., by focusing visual attention on the to-be-remembered location or locations). Next, if we turn to the issue of maintaining object representations in working memory, a significant number of studies suggests that observers can utilize cues presented during a memory retention interval to improve performance in a working memory demanding task (Averbach \& Coriel, 1961; Griffin \& Nobre, 2003; Landman et al., 2004; Makovski \& Jiang, 2007; Makovski et al., 2008; Matsukura et al., 2007; Olivers, Meijer, \& Theeuwes, 2006; Schmidt et al., 2002; Sligte, Scholte, \& Lamme, 2008; Sperling, 1960; Woodman et al., 2003). A reasonable interpretation of these findings is that the cues enable a limited-capacity rehearsal mechanism to be focused on just the task-relevant objects following the cue, resulting in higher fidelity representations of those items compared to when all of the objects must be maintained. Several of these studies interpret the cue benefits as being due to visual attention selecting the cued objects during the retention interval, akin to an object-based version of the attentional rehearsal strategy proposed for spatial locations (Awh \& Jonides, 2001). For example, Griffin and Nobre (2003) used pre-cues (i.e., cues presented prior to the memory array) and retro-cues (i.e., cues presented during the memory retention interval) to orient participants to the locations of specific objects during a change-detection task. They found reaction time (RT) and accuracy improvements following retro-cues that were equivalent to those for pre-cues compared to baseline trials with no cues. They interpreted the retro-cue benefits as evidence that we can shift attention to internal representations (i.e., in working memory).

The evidence described above is fairly clear in supporting the view that retention-interval cues indicating which items participants will be soon tested on improve performance in a change-detection task, however, the interpretation of these findings is not definitively 
settled. Specifically, it is possible that these retention-interval cues boost behavioral accuracy by reducing uncertainty about which of the multiple items in memory would be tested (e.g., Ankrum \& Palmer, 1991) and not how information is held in visual working memory. According to such an account, the representations held in memory are the same whether or not a cue is presented during the retention interval, but change-detection performance is superior following a cue because the mechanism which determines whether each item is the same or different between the memory representations and the test array can be allocated to just the relevant elements (Makovski, Watson, Koutstaal, \& Jiang, 2010). Thus, one of the goals of the present study was to evaluate this alternative explanation of the benefits of retention-interval cues using converging evidence.

It can be difficult to distinguish between competing theoretical accounts based on behavioral evidence alone. This is because it is possible for different models to mimic one another and account for the same behavioral phenomena in different ways (e.g., Logan, 2004). To overcome this problem, we will also use converging evidence from electrophysiological measurements in Experiment 3 to understand how different types of cues during memory retention intervals benefit memory-task performance. Specifically, in Experiment 3 we used event-related potentials (ERPs) to directly measure visual working memory maintenance mechanisms following cues presented during the retention interval to remember or forget a subset of the available information. This type of electrophysiological evidence allowed us to discriminate between competing explanations of the behavioral effects from the different theoretical perspectives described above.

A notable challenge to the theoretical proposals of a limited-capacity mechanism that aids visual working memory maintenance by rehearsing object representations is that empirical evidence for such maintenance has not been consistently obtained. Washburn and Astur (1998) conducted a series of experiments to test the hypothesis that there is active rehearsal of information in visual working memory using both human participants and macaque monkeys. Monkeys were used because they have visual working memory abilities comparable to humans, but lack the ability to verbalize visual information like humans (Baddeley, 1986). In several of their experiments, Washburn and Astur (1998) used dualtask paradigms with the goal of interfering with the hypothesized active maintenance process. They found that performance of the visual working memory tasks was not hindered by the concurrent tasks. In Experiment 3 of their paper, they used a directed-forgetting procedure in which cues instructed the primates to forget information on a subset of trials. They hypothesized that if rehearsal could be focused on just the task-relevant information in visual working memory, then when a cue indicated that an item could be forgotten, memory for that item should be worse and memory for the uncued items (i.e., the ones that were likely to be tested) better compared to trials without these cues. However, Washburn and Astur (1998) found that memory for the items that were cued to be forgotten was not different than a baseline condition that did not require the stimulus elements to be stored in memory. This study concluded that selective rehearsal of task-relevant information in visual working memory does not need to occur to account for the findings in working memory demanding tasks. When we consider all of the available evidence, there seems to be inconsistent empirical support for the idea that cues presented during retention intervals can be used to guide maintenance of the most relevant information.

In this study, we begin by addressing the inconsistent results obtained from paradigms using cued to remember versus cues to forget items held in visual working memory. We then discuss how we used ERP measures of visual working memory maintenance to distinguish between different explanations of the behavioral effects following the presentation of cues to remember or forget specific items held in visual working memory. 


\section{Experiment 1}

Our goal was to test the hypothesis that retention-interval cues change how objects are actively rehearsed in visual working memory by a limited-capacity mechanism. Given this capacity limit, if we have to spread our limited ability to rehearse across six objects, then our ability to accurately maintain each object representation should be worse compared to a situation where we can focus the rehearsal mechanism on half of these objects after all six are encoded into memory. To test this, we presented observers with retention-interval cues on a subset of trials that should allow rehearsal to be dedicated to just the task-relevant information of the larger set of elements stored in visual working memory.

Several features of the experimental design deserve note. Observers performed a changedetection task in which they began each trial needing to remember six colored squares. We chose this set size because six objects are sufficient to heavily tax the average participant's visual working memory capacity (Vogel et al., 2001) and reveal any cuing benefit if it exists. Throughout each trial, the participants concurrently performed an articulatory suppression task to prevent verbal recoding of the visual stimuli (Baddeley, 1986; Besner, Davies, \& Daniels, 1981) and to rule out contributions from rehearsal of the information in verbal working memory (e.g., Proctor, 1983). On half of the trials, participants were shown cues during the retention interval that indicated which subset of objects would appear during test. The test arrays on all trials probed memory for just one group of three items. This made it so that the number of decisions and responses that needed to be made on both cued and uncued trials were identical. In other words, the potential cue benefits could not be accounted for by proposing that cues reduced decision noise (Wilken \& Ma, 2004)

As shown in Figure 1, the objects were presented in two arrays of three objects with ample time for these objects to be consolidated into visual working memory between arrays (Vogel, Woodman, \& Luck, 2006). Two groups of items were presented at different times to enhance segregation of the groups of items (Palmer, 1999) and make the meaning of a presented cue unambiguous (Doll, 1969). In doing so, we expected to find a recency effect due to superior memory for the second array of stimuli, regardless of whether or not the cues influenced performance.

If a limited-capacity rehearsal process exists, then the presentation of cues should allow for this mechanism to be focused on just the task-relevant representations already in visual working memory. That is, when cued, the maintenance mechanism in visual working memory should be focused on the task-relevant subset of the stored information (i.e., three objects). Memory for the selected representations should be better compared to when maintenance is distributed across all the representations in visual working memory (i.e., six objects). Unlike Washburn and Astur (1998), we purposely refrained from testing participants on the information they were told to forget. By doing so, we predicted that observers would make use of these reliable cues and only rehearse the task-relevant information, if that is possible. As a result, observers should have superior change-detection performance following cues that afford focused rehearsal compared to uncued-baseline trials.

In Experiment 1, we also compared the effectiveness of two types of cues in different conditions. Figure 1 shows that a cue in the directed-remembering condition indicated the subset of information on which observers would be tested. Conversely, cues in the directedforgetting condition indicated what information was irrelevant because the other subset of objects would always be tested. This comparison allowed us to test another possible explanation of the previous findings in the literature. It is possible that previous evidence for rehearsal during memory-retention intervals was not obtained using directed-forgetting cues 
because directed-forgetting cues cannot be effectively used to focus maintenance on the task-relevant object representations in visual working memory (i.e., Washburn \& Astur, 1998), unlike the robust benefits shown with cues to remember (e.g., Griffin \& Nobre, 2003). If this is the case, then we predicted that we would find superior change-detection performance following cues to remember relative to performance on uncued-baseline trials, but not for cues to forget.

\section{Method}

Participants-Thirty volunteers from the Vanderbilt University community participated in this experiment for pay or partial fulfillment of a course requirement. Each participant reported having normal or corrected-to-normal vision, was not color blind, and provided informed consent before any experimental procedures began.

Stimuli-The stimuli were presented on a gray background $\left(8.20 \mathrm{~cd} / \mathrm{m}^{2}\right)$ at two eccentricities from the fixation point (a black plus sign, $0.2^{\circ} \times 0.2^{\circ},<0.01 \mathrm{~cd} / \mathrm{m}^{2}$ ) in the center of the monitor. The outer annulus centered stimuli at approximately $6.0^{\circ}$ from fixation and the inner annulus was centered $1.8^{\circ}$ from fixation. The memory stimuli were presented in groups of three squares at the same eccentricity filling three of twelve possible locations similar to a clock face. The memory stimuli were colored squares (each $1.25^{\circ} \times$ $1.25^{\circ}$ ). The color of each square was randomly selected, with replacement, from a set of seven colors: white $\left(92.46 \mathrm{~cd} / \mathrm{m}^{2}\right)$, red $\left(\mathrm{x}=.642, \mathrm{y}=.327 ; 22.62 \mathrm{~cd} / \mathrm{m}^{2}\right)$, blue $(\mathrm{x}=.152, \mathrm{y}=$. $\left.067 ; 9.66 \mathrm{~cd} / \mathrm{m}^{2}\right)$, green $\left(\mathrm{x}=.318, \mathrm{y}=.569 ; 64.99 \mathrm{~cd} / \mathrm{m}^{2}\right)$, black $\left(<0.01 \mathrm{~cd} / \mathrm{m}^{2}\right)$, yellow $(\mathrm{x}=$. $\left.478, y=.452 ; 65.23 \mathrm{~cd} / \mathrm{m}^{2}\right)$, and purple $\left(\mathrm{x}=.304, \mathrm{y}=.149 ; 7.04 \mathrm{~cd} / \mathrm{m}^{2}\right)$. The articulatory suppression stimuli were strings of four white letters or numbers (i.e., "a,b,c,d", "1,2,3,4", "w,x,y,z", or " $6,7,8,9$ "; each letter was approximately $1^{\circ} \times 1.4^{\circ}$ and the string was centered at fixation). The cue stimuli were the words "in" or "out" presented in white san serif font $\left(92.46 \mathrm{~cd} / \mathrm{m}^{2}\right.$, approximately $\left.1^{\circ} \times 1.4^{\circ}\right)$ at the center of the monitor, briefly replacing the fixation point.

Procedure-Each trial began with a memory-sample array of three items that was presented for 100-ms, followed by a 900-ms blank delay period, then a second 100-ms memory-sample array of three items, and a retention interval of 2000-ms on uncued-baseline trials. Finally, a memory-test array was presented for 5000-ms or until the observer made a response.

Fifty percent of the trials included a cue during the retention interval and these were randomly interleaved with uncued-baseline trials. During the trials with cues, the word "in" or "out" appeared for 500-ms beginning 250-ms after the offset of the second memory sample array. Following this cue, there was an additional 1250-ms retention interval. Thus, the interval between the offset of the second memory-sample array and the onset of the test array was always 2000-ms. On uncued-baseline trials, the memory-test array randomly probed subjects' memory for either the inner or outer array of three items with equal probability. As a result, every test array contained one set of three items regardless of whether a cue was presented or not. Participants indicated whether the test array was identical to the memory array presented at that eccentricity (probability $=0.5$ ) or whether the color of one of the items had changed (probability $=0.5$ ). Participants used the " $x$ " key to indicate that the test array was identical to the probed sample array and the " $\mathrm{z}$ " key to indicate it was different. Instructions stressed the accuracy of the response on each trial.

Participants were randomly assigned to either the directed-remembering condition or directed-forgetting condition. This was done to prevent misinterpretations of the meaning of the identical cues that could occur in a within-subjects design and could reduce or eliminate 
potential effects. The directed-remembering condition and the directed-forgetting condition were identical except for the meaning of the cues. In the directed-remembering condition, the cues indicated to the participants which array of squares they would need to remember at test (e.g., the inner squares would be tested when the cue was "in"). In the directed forgetting-condition, these same cues had the opposite connotation and instructed participants to forget one array of squares (e.g., the squares presented at the outer eccentricity would be tested when the cue was "in"). Aside from the test array that followed each cue, the conditions were identical in appearance. The order of inner and outer array presentation was counterbalanced across observers within each condition.

During all trials, participants were instructed to perform an articulatory suppression task to prevent verbal recoding and verbal rehearsal of the visual stimuli (Besner et al., 1981). Before the start of each block, participants were shown the string of characters to recite at a rate of 3-4 characters per second and these verbal responses were recorded to verify compliance. In both conditions, the trials were presented in four blocks of 48 trials each. Participants were allowed to take a break in between blocks for as long as they wanted. Each participant performed 12 practice trials to become familiar with the task before beginning the experimental trials.

\section{Results}

As shown in Figure 2, change-detection performance on the uncued-baseline trials was essentially identical in both conditions. Most importantly, change-detection accuracy was higher when either type of cue was provided.

Mean accuracy was analyzed using an analysis of variance (ANOVA) with the betweensubjects factor of experimental condition (directed forgetting versus directed remembering) and the within-subjects factors of cue presence (cue versus no cue), and array tested (first versus second array of objects shown). The ANOVA yielded a significant main effect of cue presence, $F(1,58)=80.84, p<0.001$, and a main effect of array presentation, $F(1,58)=$ 90.39, $p<0.001$. As shown in Figure 3, the effect of array tested was due to performance being higher when the second array was tested, as expected $(73.5 \%$ versus $86.0 \%$ in directed-remembering condition and $74.6 \%$ versus $84.1 \%$ in directed-forgetting condition, for tests of the first and second arrays, respectively). There was also a significant interaction of condition $\mathrm{X}$ cue presence, $F(1,58)=6.88, p<0.05$, due to the cue benefit being larger in the directed-remembering condition (a $9.0 \%$ difference) than in the directed-forgetting condition (a $4.9 \%$ difference). Follow-up tests within each condition showed that the effect of cue presence was significant in both of the remember and forget conditions, $F(1,29)=$ $68.05, p<0.001$ and $F(1,29)=20.10, p<0.001$, for directed-remembering and directedforgetting conditions, respectively). Furthermore, cue presence had a significant effect for both the first and second array in both experimental conditions ( $p s<0.01)$.

\section{Discussion}

In Experiment 1, we found that change-detection performance benefited from directedforgetting cues. Cues to forget did not increase accuracy as much as cues to remember, but significantly more than no cue at all. This finding nicely parallels previous research comparing cues to remember with cues to forget information in verbal working memory (Huang \& Pashler, 2007; Lee, Lee, \& Tsai, 2007). The results of Experiment 1 support the predictions of an account in which a limited-capacity mechanism enables the maintenance of information in visual working memory. As such, our findings are generally consistent with existing theoretical proposals that a limited-capacity mechanism rehearses object representations in visual working memory (e.g., Baddeley \& Logie, 1999) or for selectively 
discarding some of the contents of visual working memory to allow maintenance to be focused on the remaining representations (Duncan \& Humphreys, 1989).

We note that the findings from Experiment 1 are contrary to the directed-forgetting findings of Washburn and Astur (1998). However, we hypothesize that this difference may have been due to the fact that the items that were cued to be forgotten were not truly task irrelevant in that previous study. That is, the cues to forget did not lead to evidence of selective rehearsal because observers were presented with invalid-forget cues on $10 \%$ of the trials. On these trials, a cue indicated that items could be forgotten, but then memory was tested for these items. In this case, the ineffectiveness of the forget cues may be attributed to the fact that there was able reason to remember all information, even if the information had previously been cued to be forgotten (also suggested by Washburn \& Astur, 1998, pp. 283). In the next experiment, we tested the hypothesis that when cues to forget items in visual working memory are not consistently reliable that they do benefit memory-task performance.

\section{Experiment 2}

In Experiment 1, we used only valid cues to determine if we could observe improved change detection compared to performance from uncued-baseline trials. However, in Experiment 2 we wanted to directly test our hypothesis that the inclusion of invalid trials in the directedforgetting paradigm might lead participants to try to remember all of the items rather than using the cue (as may have occurred in Washburn and Astur, 1998). This is especially likely if a cue is considered unreliable or difficult to understand (Doll, 1969). Experiment 2 was designed to test the benefits of an unreliable cue as compared to a reliable cue. Thus, we compared change-detection performance in a 100\%-valid condition, as in Experiment 1, with a 90\%-valid condition, like that of Washburn and Astur (1998), which included 10\% invalid directed-forgetting cues (i.e., trials in which we tested participants on items that they were cued to forget). We attribute the success of the directed-forgetting cues in Experiment 1 to the fact that the cues were always $100 \%$ valid. If our assumption is correct that the invalid trials cause observers to disregard the directed-forgetting cues, then we predict that in the $90 \%$-valid condition (i.e., with $10 \%$ invalid cues) memory performance following a cue will be no different that of uncued-baseline trials.

\section{Method}

Participants-Participants were a new group of 30 volunteers from the same pool who provided informed consent prior to the beginning of the experiment.

Stimuli-The stimuli were identical to those used in Experiment 1 with the exception that they were presented in two groups at the left and right of fixation (as shown in Figure 4). For each lateral array of three objects there were six possible locations with a minimum distance of $0.55^{\circ}$ from each other and $1.8^{\circ}$ from fixation. The use of different array configurations in Experiment 2 allowed us to generalize the findings of Experiment 1 in the directedforgetting condition to a variety of spatial cues indicating the task-relevant objects.

Procedure-The procedure was identical to Experiment 1 except as noted. The order of left and right array presentations was counterbalanced across participants within each condition. Observers used a gamepad rather than the keyboard used in Experiment 1 to make their responses. Participants pressed one button to indicate that the sample and test arrays were identical ("same") and a different button to indicate that a color of one square in the array had changed (“different”).

Experiment 2 had two directed-forgetting conditions. Participants were randomly assigned to either the $100 \%$-valid or $90 \%$-valid condition. In the $100 \%$-valid condition, the cues 
accurately indicated which group of items would be presented in the test array on $100 \%$ of trials. In the $90 \%$-valid condition, $10 \%$ of the cues were invalid and $90 \%$ were valid. On an invalid-cue trial the probe would indicate that an array of three items could be forgotten (e.g., on the left), however, that array would then be tested at the end of the retention interval (e.g., a left hemifield test array). For both groups, we instructed participants that the cue would be the best indicator of what to hold in memory until the test array was presented.

\section{Results}

Figure 5 shows the change-detection accuracy in the $90 \%$-valid and $100 \%$-valid conditions. Uncued-baseline accuracy for both the $90 \%$-valid and the $100 \%$-valid conditions was similar. In the $90 \%$-valid condition, change-detection accuracy on validly cued trials was essentially identical to performance on the uncued-baseline trials. In contrast, changedetection accuracy was higher during the cued trials in the $100 \%$-valid condition than in the baseline trials. Thus, the inclusion of the $100 \%$-valid condition allowed us to replicate the critical directed-forgetting findings of Experiment 1 with a different group of observers and simultaneously showed how the inclusion of invalid trials eliminates these effects.

Accuracy data were analyzed with an ANOVA with the between-subjects factor of experimental condition (100\%-valid versus $90 \%$-valid cues) and the within-subjects factors of cue presence (cue versus no cue) and array tested (first versus second array of objects shown). ${ }^{1}$ This yielded main effects of array presentation, $F(1,58)=57.61, p<0.001$, and a nearly significant interaction of experimental condition $\mathrm{X}$ cue presence, $F(1,58)=3.8, p=$ 0.056. Planned comparisons showed that in the $100 \%$-valid condition, change-detection accuracy was significantly higher when a cue was presented than on the uncued-baseline trials, $F(1,29)=4.30, p<0.05$. In the $90 \%$-valid condition, performance on cued trials was not significantly different than baseline trials, $F(1,29)=0.66, p>0.40$. We also found a significant effect of second array presentation in both conditions, $F(1,29)=29.31, p<0.001$, in the $90 \%$-valid condition and, $F(1,29)=28.53, p<0.001$, in the $100 \%$-valid condition. Table 1 shows that performance was better in both conditions when memory for the second set of items was tested (this includes baseline and cued trials). As in Experiment 1, this is evidence for the expected recency effect in this two-array paradigm. However, only the $100 \%$-valid condition showed an increase for cued second array trials compared to baseline trials in which the second array was tested.

In our next analysis, we examined the nature of the cuing effects across the experimental session. We thought it might be possible that observers in the $90 \%$-valid condition initially made use of the cues to forget and show a cuing benefit early in the experimental session (i.e., until they encounter their first few invalid cues). However, Figure 6A shows that this pattern was not observed across blocks of trials. The cuing effect was absent from the $90 \%-$ valid condition even in the earliest block and the results from the $100 \%$-valid condition show that the cuing benefit grew fairly slowly across the experiment. An ANOVA with the between-subjects factor of experimental condition (100\%-valid versus $90 \%$-valid) and the within-subjects factors of cue presence (present versus absent) and trial block (1, 2, 3, or 4) yielded a significant main effect of block, $F(3,174)=3.95, p<0.01$, and significant interactions of experimental condition $\mathrm{X}$ block, $F(3,174)=3.01, p<0.05$, and experimental condition $\mathrm{X}$ cue presence, $F(1,58)=5.13, p<0.05$. However, there was not a significant

\footnotetext{
${ }^{1}$ The results for the $90 \%$-valid condition in Experiment 2 were analyzed using data from both the valid and invalid trials to maximize power. However, a separate analysis of just the valid trials was also carried out to confirm that the potency of the valid cues was reduced by the possibility of the invalid cues and not the inclusion of these trials themselves. We observed the same pattern of effects when comparing magnitudes of cue benefits. Even without the invalid trials included in the analysis, cues were more beneficial during the $100 \%$-valid condition ( $71.4 \%$ valid cues versus $68.4 \%$ baseline, $p<0.05)$ than in the $90 \%$-valid condition ( $70.5 \%$ valid cues versus $71.1 \%$ baseline, $p=.78$ ).
} 
three-way interaction, $F(3,174)=0.44, p>0.70$. Follow-up tests within each condition (i.e., $100 \%$-valid versus $90 \%$-valid) showed that in the $100 \%$-valid condition there was a main effect of block, $F(3,87)=4.01, p<0.05$, and a marginally significant main effect of cue presence, $F(1,29)=3.78, p=0.06$. The $90 \%$-valid condition also yielded a main effect of block, $F(3,87)=3.01, p<0.05$, but had no other significant main effects or interactions.

To determine whether this slow progression of cue utilization we observed in Experiment 2 is typical in visual working memory cuing paradigms we reexamined the data from Experiment 1 using this same block-by-block analysis. Figure 6B shows that participants in Experiment 1 also initially exhibited a smaller change-detection performance advantage following cues at the beginning of the experimental session and that the effectiveness of the cues grew in size across trials, particularly in the directed-forgetting condition. The ANOVA yielded a significant main effect of block, $F(3,174)=6.26, p<0.001$, and a significant interaction of experimental condition $\mathrm{X}$ cue presence, $F(1,58)=6.88, p<0.05$. Follow-up tests showed that there were main effects of cue presence $(p s<0.001)$ and block $(p s<0.05)$ in both the direct-remember and the direct-forget condition. However, neither experimental condition yielded interactions of cue presence by block.

\section{Discussion}

In Experiment 2, we compared the effectiveness of directed-forgetting cues in a condition in which invalid cues were possible, although infrequent (i.e., $10 \%$ of cuing trials), to a condition in which all cues to forget were valid, as in Experiment 1 . We found that when invalid cues were possible, change-detection accuracy did not differ between trials in which a cue was provided and those when no cue was presented. Indeed, we found a trend toward the presentation of the cues in the $90 \%$-valid condition causing interference with maintenance and reducing change-detection accuracy. Observers appeared to disregard the probabilistic information carried by the cue and tried to remember all of the objects given they knew they might be tested on either subset. During debriefing, several observers reported that they explicitly adopted this strategy after realizing that they might need to know the identities of the objects they were cued to forget.

These findings demonstrate that the inclusion of invalid directed-forgetting cues in a visual working memory task can cause observers to disregard the information presented by the cue even when it accurately predicts task relevance $90 \%$ of the time. Our human participants appear to have behaved like the monkeys in the study of Washburn and Astur (1998). Because they experienced invalid cues, they continued to maintain all of the items in memory following a cue to forget a subset of the objects.

The analysis of performance across the experimental session showed that the effectiveness of the cue increased across time. This observation is consistent with the interpretation that participants default mode of processing is to rehearse all of the items shown. The utilization of the cue to forget some of the information appears to take the form of a strategic operation that is implemented more effectively with additional practice and experience. These findings emphasize the importance of strategic factors in determining whether someone will maintain a piece of information in visual working memory and converge with the classic literature on the strategic nature of rehearsal in temporary memory (Brown, 1958; Cermak, 1972).

Indeed, the relatively slow growth of the cuing effect across trial blocks is reminiscent of the time course of changes in performance during other tasks that are thought to tap into the executive control of information in working memory, like the Wisconsin Card Sorting Test and the Tower of Hanoi task (Miyake et al., 2000). This is also consistent with evidence that visuo-spatial working memory abilities are related to executive functions (such as information updating and monitoring; Miyake, Friedman, Rettinger, Shah, \& Hegarty, 2001) 
that are important in the paradigm used here where the set of information that is being maintained needs to be updated in response to a cue.

Our block-by-block analyses showed a pattern of findings that are also relevant for a longstanding debate over whether it is possible to selectively forget information or whether it is really due to the prioritization of other information for access to maintenance mechanisms. If the mechanism underlying the benefit of the cues was akin to attentional selection prioritizing the task-relevant items in memory (Makovski \& Jiang, 2007; Makovski et al., 2008), then we would expect the benefit of the cues to emerge quickly given that spatial cuing of visual-spatial attention has a rapid time course within a trial and does not appear to rely heavily upon extensive learning of the task contingencies (Posner \& Cohen, 1984). However, it is clear that in the context of the working memory task we used here that any mechanism underlying performance must be relatively slow to engage with experience performing the task. Given the observed time course, it would seem possible to conclude that the use of directed-forgetting cues does not rely upon a selection mechanism to prioritize the representations stored, but instead a slower acting process such as an additional inhibition operation or learning to remap the meaning of the forget cues to prioritize the other set of representations. Although this is one set of explanations, other evidence makes it difficult to draw definitive conclusions based on these findings. For example, recent work has shown that some attentional cuing effects that prioritize new perceptual inputs for preferential processing appear to rely heavily upon learning and long-term memory of the task contingencies (Cosman \& Vecera, 2011). Thus, it is clear that the findings of our blockwise analysis shows that the directed-forgetting effects develop across fairly long intervals of time and it remains to be seen whether this time course is too slow to be accounted for by other mechanisms of selection or prioritization. We will return to this issue of the speed with which the cues can be utilized in Experiment 3.

\section{Experiment 3}

In our previous experiments, it is possible that the modest behavioral benefits that followed the presentation of cues during the retention intervals were not due to changes in how information was maintained. Specifically, it is possible that the cues to forget or remember subsets of the information really changed the operation of decision processes. Perhaps orienting decision mechanisms to the items the cues indicated would be relevant in the test array improved performance while the maintenance of the information in visual working memory was identical whether or not a cue was presented.

In Experiment 3, our goal was to use ERPs to directly test the hypothesis that the cue benefits we observed in the previous experiments were due to the focusing of maintenance mechanisms on the task-relevant information. To this end, we measured the contralateraldelay activity (or CDA) during a change-detection paradigm similar to that used in Experiment 2 except that the three items in the right and the left hemifield were presented simultaneously in one bilateral, six-item array to prevent the arrays from eliciting lateralized sensory potentials. We focused on the CDA component of the participants ERP waveforms because this component is believed to directly measure the maintenance of task-relevant information in visual working memory (Vogel \& Machizawa, 2004; Vogel, McCollough, \& Machizawa, 2005; Woodman \& Vogel, 2008). When observers are cued to remember a set of items in one visual field (e.g., left of fixation) prior to the onset of the memory array, a sustained contralateral negativity is found following the onset of the memory array and continuing until memory is tested (e.g., over the right hemisphere relative to the left hemisphere). This sustained contralateral negativity shows a systematic increase in amplitude up to the point that an individual participant's visual working memory is filled (Vogel \& Machizawa, 2004). Thus, the CDA is an ideal tool for testing the hypothesis that 
the behavioral benefits of the cues presented in the previous experiments were a result of focusing maintenance on the task-relevant items indicated by the cues.

On the uncued-baseline trials in the previous experiments we reasoned that maintenance would be distributed across all of the memoranda in the arrays. In terms of the CDA, this would result in the three memoranda in both the left and right hemifields eliciting negative potentials. As a result, we expected that we would observe similar potentials across both hemispheres on the uncued-baseline trials. If cues to remember a subset of the items already stored in visual working memory are effective because they focus maintenance on the cued items, then we should see that a CDA emerges contralateral to those items after the cue. Similarly, if directed-forgetting cues result in superior behavioral performance because observers focus their limited-capacity maintenance mechanism on just the task-relevant information in visual working memory, then following a cue indicating that the information in one hemifield can be forgotten, we should observe a CDA emerge contralateral to the uncued items (i.e., the items that still need to be remembered). Thus, we can use the CDA to test the hypothesis that the benefit of cues to remember and forget have a similar underlying cause, but potentially different temporal onsets of this focused maintenance, accounting for the superior performance following remember cues compared to forget cues.

Alternatively, the retention-interval cues to forget or remember a subset of the items in Experiments 1 and 2 may have caused the modest improvements in change-detection accuracy by focusing mechanisms unrelated to visual working memory maintenance on the relevant items. In particular, if the retro or retention-interval cues used here and in previous work changed decision-related processes (e.g., Griffin \& Nobre, 2003), then we predicted that the ERP differences between cued and uncued trials would emerge following the presentation of the test array, as decision were limited to just the cued set. We expected that such effects would reveal themselves as larger and earlier lateralized effects following the presentation of the test array (e.g., Hyun, Woodman, Vogel, Hollingworth, \& Luck, 2009) without the CDA effects during the retention interval.

\section{Method}

Participants-The participants were a new group of 24 volunteers, 12 in the directedforgetting condition and 12 in the directed-remembering condition. Volunteers were paid for their participation. All had normal color vision and acuity (or corrected to normal), in addition to no history of neurological problems. They provided informed consent prior to the start of the experimental procedures.

Stimuli-Figure 7A shows that the stimuli were identical to those used in Experiment 2 with the exception that all six differently colored squares were presented at once. Three squares appeared to the left of the horizontal meridian and three squares to the right. For each lateral array of three objects there were six possible locations with a minimum distance of $0.55^{\circ}$ from each other and $1.8^{\circ}$ from fixation. We verified in a pilot experiment $(\mathrm{N}=18)$ that we could observe hemifield cuing effects from memory arrays in which 6 items were presented simultaneously (three in the left and three in the right hemifield). We were concerned that the simultaneous presentation of six items in one array would lead to different performance than when two, three-item arrays were shown (Ihssen, Linden, \& Shapiro, 2010). However, the size of the hemifield cuing effects (i.e., directed forgetting "left" versus "right" cues) and the mean accuracy in the baseline trials when all six items were presented simultaneously did not significantly differ from that of the $100 \%$-valid condition of Experiment 2 in which two, 3-item arrays were presented, $F s<1.0$ (contrary to Ihssen, Linden, \& Shapiro, 2010). 
Procedure-The six-item memory array was presented for $100 \mathrm{~ms}$. On uncued-baseline trials, this array was followed by a 2200-ms retention interval before the presentation of a three-item test array. Participants needed to remember three items on both the left and right side of the array unless a cue indicated that they could forget the items in one hemifield. On trials with a directed-forgetting cue (i.e., in the directed-forgetting condition), the 100-ms memory array was followed by a $250 \mathrm{~ms}$ blank interval, then a $500-\mathrm{ms}$ cue (i.e., "left" or "right"), and another $1450 \mathrm{~ms}$ blank interval before the test array of three items was presented. Cue trials in the directed-remember condition were identical except that the meaning of the cue was reversed (e.g., a the "left" meant that participants need to continue to remember the items presented in the left hemifield).

$100 \%$-valid directed-forgetting cues were presented on $50 \%$ of trials with all of the participants. Participants were instructed as to the meaning of the cues and familiarized with the task during 12 practice trials before 16 blocks of 48 experimental trials each.

ERP Recording and Analysis-The electroencephalogram (EEG) was recorded from tin electrodes held on the scalp by an elastic cap (Electrocap International, Eaton, OH). A subset of the International 10/20 System sites were used (Fz, Cz, Pz, F3, F4, C3, C4, P3, P4, PO3, PO4, T3, T4, T5, T6, O1, and O2) as well as the nonstandard sites OL (halfway between $\mathrm{O} 1$ and T5) and $\mathrm{OR}$ (halfway between $\mathrm{O} 2$ and T6). The right mastoid electrode served as the online reference for these active electrode sites. The signals were re-referenced offline to the average of the left and the right mastoids (Nunez, 1981). The electrooculogram (EOG) was recorded by placing electrodes $1 \mathrm{~cm}$ lateral to the external canthi to measure horizontal eye movements and by placing an electrode beneath the left eye, referenced to the right mastoid, to measure vertical eye movements and blinks. The EEG and EOG were amplified by an SA Instrumentation amplifier with a gain of 20,000 and a bandpass of 0.01-100 Hz. The amplified signals were digitized at $250 \mathrm{~Hz}$ by a PC-compatible computer and averaged offline. Trials accompanied by incorrect behavioral responses or ocular or myogenic artifacts were excluded from the averages.

We used a two-step procedure for ocular artifact rejection that has been described previously (Woodman \& Luck, 2003). Participants ERP data was considered if they could refrain from making detectable eye movements on a majority of the trials, as judged online by the experimenter. Trials with large eye movements were rejected prior to averaging, and averaged horizontal EOG (HEOG) waveforms were used to reject any subjects with significant residual eye movements. Then, participants were replaced if they had either greater than $30 \%$ of individual trials rejected or any residual systematic eye momovement that resulted in HEOG voltage deflections greater than $3.2 \mu \mathrm{V}$, corresponding to an ocular deviation of $\pm 0.1^{\circ}$ (Nunez, 1981). This procedure led to the rejection of an average of $14.4 \%$ of trials per participant (with a single participant maximum of 26.4\%) and required us to replace a total 10 participants from both conditions due to excessive eye movements.

Contralateral-delay activity (i.e., the CDA) was measured as the difference between electrode sites contralateral and ipsilateral to the items that would be tested during the upcoming test array of that trial. On trials with a cue, the cues were $100 \%$ valid so observers knew whether the items on the left or the right of the array would be tested on the trial. We measured the amplitude of the CDA during a time window 500-2400 ms after the onset of the memory array. Analyses of variance (ANOVAs) were used for all statistical tests, and $p$ values were adjusted using the Greenhouse-Geisser epsilon correction for nonsphericity (Jennings \& Wood, 1976). 


\section{Results}

Behavioral-As shown in Figure 7B, change-detection accuracy on trials with directedforgetting cues was significantly higher than on uncued-baseline trials (a 5.4\% difference), $F(1,11)=16.29, p<0.01$. Similarly, in the directed-remembering condition performance on cued trials was better compared to baseline trials (a 9.3\% difference), $F(1,11)=84.13, p<$ 0.01 . The same pattern was observed in the between-group analysis where cues yielded a significant main effect, $F(1,22)=76.75, p<0.01$. We also found a significant interaction of experimental condition X cue, $F(1,22)=5.44, p<0.05$, due to the behavioral advantage of the cue being larger in the directed-remembering condition. These behavioral findings serve to replicate those of the previous experiments.

ERPs-Figure 8 shows the ERPs recorded during uncued-baseline trials and trials with cues in the directed-forgetting and directed remembering conditions, time locked to the onset of the six object memory array. The memory and test arrays elicited large ERP components related to sensory and perceptual processing (i.e., the P1 and N1) on both trial types. The unilateral test arrays elicited the canonical hemispheric asymmetries in the P1 and $\mathrm{N} 1$ followed by a sustained contralateral negativity riding on the large P3 waveform, presumably related to comparing the test array to the memory representation (e.g., Hyun et al., 2009).

The most critical portion of the waveforms occurred during the memory retention intervals. On the uncued-baseline trials, when we required participants to remember all six items from the memory array, we found the expected pattern of results in which the voltage was similar across both hemispheres (i.e., contralateral and ipsilateral to the items that were ultimately tested). However, when a directed-forgetting cue indicated that observers could forget all of the items in one hemifield, we found that a clear negativity emerged after the cue contralateral to the items that the cue indicated were still necessary to remember (approximately $100 \mathrm{~ms}$ after cue offset or $1000 \mathrm{~ms}$ after the memory array onset). We observed a similar pattern on cued trials of the directed-remembering condition, indicating that the benefits of both types of cues are due to changes in how information is maintained during the retention interval, following the cues. However, the CDA following cue to remember onsets earlier and is larger in amplitude than the CDA following a cue to forget. These patterns of electrophysiological results are consistent with what we expected if participants were using the cues to focus maintenance on just the task-relevant items.

To verify the statistical significance of these observations, we entered the mean ERP voltages measured from 500-2400 ms after the onset of the memory array into an ANOVA using the between-subjects factor of condition (directed forgetting versus directed remembering) and the within-subject factors of trial type (uncued baseline versus cues), contralaterality (contralateral versus ispilateral to the tested items), hemisphere (left versus right), and electrode site (PO3/4, P3/4, O1/2, OL/R, versus T5/6). Of primary importance, in this between-subjects ANOVA we did not observe a significant main effect of condition or an interaction of condition and contralaterality, $F S>1.0$. This indicates that for both the directed-forgetting and directed-remembering trial types we observed a similar CDA relative to the task-relevant information following the cue. This ANOVA did yield significant main effects of trial type, $F(1,22)=12.84, p<0.01$, contralaterality, $F(1,22)=21.03, p<0.001$, and electrode site, $F(4,88)=9.06, p<0.001$, as well as interactions of trial type $\mathrm{X}$ contralaterality due to the CDA being present on cued trials but not baseline trials, $F(1,22)=$ $18.38, p<0.001$. The interactions of contralaterality X electrode site, $F(4,88)=6.65, p<$ 0.001 , and trial type $\mathrm{X}$ contralaterality $\mathrm{X}$ electrode site, $F(4,88)=5.82, p<0.001$, were due to the CDA being maximal at temporal-occipital electrodes. 
Next, we performed planned within condition analyses. We found a significant interaction of trial type $\mathrm{X}$ contralaterality $\mathrm{X}$ electrode site in both the directed-forgetting, $F(4,44)=3.43, p$ $<0.05$, and the directed-remembering condition, $F(4,44)=8.39, p<0.01$. These main effects were due to the voltages being more negative (particularly at T5/6 and OL/R) contralateral to the tested items on trials with cues but not uncued-baseline trials. Consistent with this, there was no significant difference between electrodes contralateral versus ipsilateral to the tested items during the retention intervals of the uncued-baseline trials in the directedforgetting condition, $F(1,11)=0.05, p>0.80$, and the directed-remembering, $F(1,11)=0.02$, $p>0.80$. However, there was a significant difference between contralateral and ipsilateral waveforms on trials with cues to forget and remember, $F(1,11)=5.38, p<0.05$, and $F(1,11)$ $=20.13, p<0.001$, respectively. No other main effects or interactions were significant in the directed-forgetting ANOVA. However, the directed-remembering ANOVA also yielded significant main effects of trial type, $F(1,11)=13.89, p<0.01$, and electrode site, $F(4,44)=$ $6.86, p<0.001$, as well as interactions of trial type $\mathrm{X}$ contralaterality, $F(1,11)=11.99, p<$ 0.01 , contralaterality $\mathrm{X}$ electrode site, $F(4,44)=8.24, p<0.001$, and condition $\mathrm{X}$ trial type $\mathrm{X}$ contralaterality, $F(4,44)=4.69, p<0.01$.

Finally, we measured the latency of the CDA following cues to remember versus cues to forget. The fractional-area latency was measured as the time point at which $50 \%$ of the area under the CDA voltage had been reached (Luck, 2005; Woodman, 2010). For this analysis, we measured the ERP waveform 500 to $2400 \mathrm{~ms}$ following the onset of the array presentation. As shown in Table 2, the $50 \%$ fractional-area latency of the CDA on cue trials is approximately $320 \mathrm{~ms}$ later in the directed-forgetting condition than in the directedremembering condition.

However, this effect did not reach significance, $F(1,22)=3.02, p=0.096$, due to the variability in this metric across participants. This trend in the onset latency being later following directed-forgetting cues and cues to remember suggests that directed forgetting cues may take longer to interpret than the less circuitous remember cues. As discussed further below this could also provide preliminary evidence for a discard operation that precedes the focusing of attention. Further study of this potential onset effect with a design focused on measuring this potential phenomenon may help to resolve some of our lingering questions about the precise temporal sequence of the mechanisms that come into play prior to the CDA related to maintaining the cued information and potentially explain the consistent behavioral benefit of remember cues over cues to forget.

\section{Discussion}

In Experiment 4 we found a pattern of results that serves to significantly constrain the process explanations underlying the behavioral benefit of directed-forgetting and remembering cues in Experiments 1-3. The presence of a CDA following both types of retention-interval cues shows that these behavioral performance benefits are accompanied by the electrophysiological signature of focusing maintenance on just the task-relevant items as indicated by the cue. In addition, we have consistently observed that directedremembering cues result in a behavioral benefit that is consistently larger than that of directed-forgetting cues. Our ERP findings suggest that our ability to more efficiently orient maintenance mechanisms to the task-relevant representations underlies this modest, but consistent, behavioral advantage of directed-remembering cues. As discussed further below, this focusing of visual working memory maintenance could be the sole act of selection underlying the behavioral benefit from cues about future task relevance, or this focusing of maintenance could follow a preceding operation in which the to-be-forgotten items are flushed from visual working memory. 
Our ERP findings do address a number of uncertainties that had remained about the effectiveness of retention-interval cues (or retro-cues). Regardless of our efforts to design Experiment 1 and 2 such that the decision demands were identical between uncued-baseline trials and cued trials (i.e., always 3 items), it is possible that the cues served to orient the decision processes and not maintenance mechanisms. That is, a class of models propose that many apparent capacity limits during tasks are easily explainable by decision noise (e.g., Shaw, 1982). These models assume that each decision (i.e., is this item the same as the one I saw in the memory array) is accompanied by some probability of making an error, such that when more items must be considered as either same or different from the first, memory array the probability of making a decision error increases. It is possible that the retro or retention-interval cues used here, and in previous studies, actually focused decision processes on only half of the items and that the cause of the modest behavioral benefits of cues was actually due to a reduction of decision noise. Indeed, previous studies have sought to minimize or eliminate decision noise while varying information load in working memory tasks by using cues that appear concurrently with the test arrays (e.g., Vogel et al., 2001). Our ERP findings demonstrate that cues presented during memory retention intervals are not simply allowing people to focus decision mechanisms on the items that are tested. ${ }^{2}$ Instead, our findings provide direct electrophysiological evidence that the cues result in visual working memory maintenance being refocused by retention-interval cues. This selection of the task-relevant subset of information (i.e., 3 items) then results in the cued items being better remembered compared to when the full set of items were maintained (i.e., 6 items).

\section{General Discussion}

In Experiment 1, we found that participants' change-detection accuracy was improved by cues that allowed them to predict with certainty which items were going to be task relevant. Cues effectively reduced the task-relevant set size after all six items had been encoded. Although both directed-remembering and directed-forgetting cues significantly improved performance, we found that the cues to remember a subset of objects were more effective than cues to forget a subset. In Experiment 2, we reconciled the findings of Experiment 1 with those of a previous report that found that directed-forgetting cues did not influence performance in a visual working memory task (Washburn \& Astur, 1998). Specifically, if it was possible that participants would be tested on the objects they were cued to forget, then the observers appeared to ignore the cues altogether. In Experiment 3, we showed that the benefits of cues to forget generalized to a subset of items presented simultaneously in a larger array. This ruled out the possibility that the findings were an artifact of the presentation procedure we used in Experiment 1 and 2 in which the to-be-remembered information was presented in two discrete arrays. Finally, in Experiment 4, we used participants' ERPs to show that working memory maintenance is refocused based on informative cues. This refocusing of maintenance results in superior memory-task performance demonstrating that the maintenance of information in visual working memory is limited in capacity. Thus, this evidence is consistent with a limited-capacity rehearsal process that is actively deployed to the most relevant information stored in visual working memory.

\footnotetext{
${ }^{2}$ We note that our ERP findings cannot rule out an explanation based on the idea that decision processes are prepared in advanced to act on certain representations. That is, it is possible that the CDA represents some broadly defined decision mechanism that tags representations for the potential comparison with test items in the future. It is unclear how such an explanation of the CDA could account for the existing results from ERP studies showing capacity limits of about 3 to 4 items and this seems to deviate qualitatively from the type of model of decision-making proposed in the literature (e.g.,Shaw, 1982). It is possible that future studies would be able to distinguish between such a decision preparation mechanism that maintains some type of tags for future decision and a true active maintenance mechanism in visual working memory, but it is also possible that these accounts mimic one another even at a neural level.
} 
We designed our experiments to rule out three straightforward alternative explanations for the findings. First, we required our participants to concurrently articulate to prevent them from verbally recoding and rehearsing the visual information (Proctor, 1983; Shaffer \& Shiffrin, 1972). Second, we required the participants to make the same number of decisions and responses on each trial, whether or not a cue was provided, so our findings are not consistent with decision-noise explanations of these cue benefits (Vogel et al., 2001; Wilken $\& \mathrm{Ma}, 2004)$. Moreover, Experiment 3 provided converging ERP evidence that the cues resulted in differential maintenance of the items compared to uncued trials. Third, our paradigm rules out the explanation that trials with a cue placed a lighter load on the limited representational capacity of visual working memory. We required the participants to encode six items into visual working memory on each trial. Only after all the items were held in memory, we then occasionally provided a cue indicating which three items they should continue to maintain. Let us assume that each subject has three objects' worth of representational storage capacity in visual working memory (estimated capacity of the participants in Experiment 1 was 3.74 objects). Given they need to store six items because a cue might not be provided, and if so, they do not know what set will be cued, they store half an object's worth of information $(6 / 3=0.5)$ about each item. If a cue later tells them that they need not maintain three of those representations, then they would still have at best half an object's worth of information about each item shown in the memory arrays. Simply dropping three of these representations does not compensate for the loss of information at the front end of storage and by the time the cue is presented, the perceptual and iconic memory representations of the items are not available to improve these representations (Averbach \& Coriel, 1961; Sperling, 1960). Thus, our empirical observations of benefits due to cues to remember or forget cannot be accounted for by verbal rehearsal, decision-noise explanations, or a simple reduced load explanation without appealing to the idea of a limited-capacity process being dedicated to the remembered information.

According to the model of Logie and Baddeley (Baddeley \& Logie, 1999; Logie, 1995), on the trials that we provided participants with cues, the inner scribe could just refresh the representations that the cue indicated would be tested at the end of the retention interval. By focusing this limited-capacity rehearsal mechanism on just the task-relevant representations they remain veridical while the unrehearsed representations are allow to decay or die in the passive visuo-spatial sketchpad (Matsukura et al., 2007; Zhang \& Luck, 2009).

Alternatively, the cue benefits we observed could be due to active forgetting operations taking place following such cues in which irrelevant object representations are discarded from visual working memory (e.g., Duncan \& Humphreys, 1989). That is, the

representations that are determined to be irrelevant following the cue might be suppressed or expelled from visual working memory (Bissett, Nee, \& Jonides, 2009; Nee \& Jonides, 2008) which then results in the refocusing of rehearsal mechanisms on the remaining representations. It is possible that some addition benefit could also be derived from this act of discarding information in terms of relieving the remaining items from the interference that would have been caused by the additional representations in working memory. These competing theoretical explanations boil down to whether the relevant objects are selected for enhanced processing or whether the irrelevant items are discarded from the visual store.

Many phenomena in the cognitive literature could be due to the enhancement of the relevant information or the suppression of the irrelevant information (Logan, 2004; Luck, 1995) and it seems that our findings show another instance in which these competing ideas can be tested. Currently, our ERP data favor the explanation that both cues to forget or remember information result in a similar refocusing of maintenance on the remaining task-relevant information. The superior behavioral performance with remember cues is paralleled by a larger CDA component suggesting that this refocusing occurs more efficiently with remember cues compared to forget cues. However, we also find a suggestion in our ERP data that the onset of the CDA following a directed-forgetting cue is slower than when the 
CDA is observable following a directed-remembering cue. If this observation is real, it is consistent with the idea that we might spend time expelling the irrelevant information following a cue to forget before refocusing maintenance, whereas only a simple refocusing is evoked by cues to remember. We hope that future experiments will be able to distinguish between this simple, more parsimonious explanation and the more sophisticated mechanism in which information is expelled prior to maintenance shifting to the remaining representations.

Although our findings consistently supported the theoretically motivated hypothesis that a limited-capacity rehearsal mechanism can be focused on just the task relevant information in visual working memory, our findings might be interpreted differently given another theoretical framework. Specifically, recent theoretical work has proposed that the storage of information in visual working memory might be made possible by the allocation of a flexible resource (e.g., Bays \& Husain, 2008). Such a model might reasonably propose that this resource can be redistributed based on new information after items have been encoded. If the result of this redistribution is that items receiving the lion's share of resources are remembered better than the same items when they equally share the resources with a larger group of representations, then this becomes an issue of terminology. We have couched our findings in the context of the modal view in the working memory literature (Miyake \& Shah, 1999) in which information in working memory is actively maintained whether it is discrete object representations, active long-term memory representations or some other representational format, however, it is possible to describe the present findings with a more mechanistically neutral and expansive term, like resources (Navon, 1984).

Are the cue benefits we observed due to selection by the same visual attention mechanisms that operate on perceptual inputs or is this capacity-limited rehearsal due to a visual working memory maintenance mechanism that is distinct from visual attention? The visuo-spatial working memory model of Logie and Baddeley (Baddeley \& Logie, 1999; Logie, 1995) describes the inner-scribe as a spatial maintenance mechanism that serves to rehearse object representations in visual working memory and is distinct from the control mechanisms of the central executive in which attention is presumed to reside. A body of existing evidence suggests that this rehearsal mechanism only operates on the contents of visual working memory and is distinct from perceptual mechanisms of selection (Owen, 1997; Woodman \& Luck, 2007; Woodman, Vogel, \& Luck, 2001). For example, maintaining a group of items in visual working memory does not interfere with the efficiency of a perceptual-attention demanding task like visual search, suggesting that perceptual attention and visual working memory rehearsal mechanisms can operate asynchronously (Woodman et al., 2001). In contrast, other theorists have proposed that maintaining information in visual working memory may rely upon the same visual attention mechanisms that operate during perceptual processing (Awh \& Jonides, 2001; Cowan, 1997). As of yet, our findings to not definitively distinguish between these two proposed rehearsal mechanisms. Our observation that the CDA is elicited following the cues to forget items is suggestive that we are seeing the effects of a visual working memory rehearsal mechanism that is different from those of perceptual mechanisms of selection (Ikkai, McCollough, \& Vogel, 2010), however, our understanding of the cognitive operations underlying each ERP waveform is a constantly evolving process (Woodman, 2010). We are hopeful that converging evidence from neurophysiological, neuropsychological, and behavioral studies will be able to determine the precise locus of the rehearsal-related effects we observed here.

\section{Acknowledgments}

We thank Jurnell Cockhren who provided programming assistance, as well as Mimi Zhao and Kristen Quinn who helped with data collection. George Alvarez, Nancy Carlisle, Jason Arita and Min-Suk Kang provided useful 
suggestions during this project. Mike Dodd, Brad Gibson, Chris Olivers and six anonymous reviewers provided invaluable comments on previous versions of this paper. This research was supported by the National Eye Institute of the National Institutes of Health (RO1-EY019882) and the National Science Foundation (BCS 09-57072, and a graduate research fellowship to M.W.).

\section{References}

Ankrum C, Palmer J. Memory for objects and parts. Perception and Psychophysics. 1991; 50(2):141156. [PubMed: 1945736]

Averbach E, Coriel AS. Short-term memory in vision. Bell System Technical Journal. 1961; 40:309328.

Awh E, Anllo-Vento L, Hillyard SA. The role of spatial selective attention in working memory for locations: Evidence from event-related potentials. Journal of Cognitive Neuroscience. 2000; 12:840-847. [PubMed: 11054925]

Awh E, Jonides J. Overlapping mechanisms of attention and spatial working memory. Trends in Cognitive Sciences. 2001; 5:119-126. [PubMed: 11239812]

Awh E, Jonides J, Reuter-Lorenz PA. Rehearsal in spatial working memory. Journal of Experimental Psychology: Human Perception \& Performance. 1998; 24(3):780-790. [PubMed: 9627416]

Baddeley, AD. Working Memory. Oxford: Clarendon; 1986.

Baddeley, AD.; Logie, RH. Working memory: The multiple component model. In: Shah, P.; Miyake, A., editors. Models of working memory. Cambridge, UK: Cambridge University Press; 1999.

Bays PM, Husain M. Dynamic shifts of limited working memory resources in human vision. Science. 2008; 321(8):851-854. [PubMed: 18687968]

Besner D, Davies J, Daniels S. Reading for meaning: The effects of concurrent articulation. Quarterly Journal of Experimental Psychology. 1981; 33A:415-437.

Bissett P, Nee DE, Jonides J. Dissociating interference-control processes between memory and response. Journal of Experimental Psychology: Learning, Memory, and Cognition. 2009; 35(5): $1306-1316$.

Brown J. Some tests of the decay theory of immediate memory. Quarterly Journal of Experimental Psychology. 1958; 10:12-21.

Cermak LS. Rehearsal strategy as a function of recall expectation. The Quarterly Journal of Experimental Psychology. 1972; 24(4):378-385.

Cowan, N. Attention and Memory. New York: Oxford University Press; 1997.

Cowan, N. An Embedded-Processes Model of working memory. In: Miyake, A.; Shah, P., editors. Models of working memory: Mechanisms of active maintenance and executive control. Vol. xx. New York: Cambridge University Press; 1999. p. 506

Cowan N. The magical number 4 in short-term memory: A reconsideration of mental storage capacity. Behavioral and Brain Sciences. 2000; 24:87-185. [PubMed: 11515286]

Doll TJ. Short-Term Retention: Preparatory Set as Covert Rehearsal. Journal of Experimental Psychology. 1969; 82(1):175-182.

Duncan J, Humphreys GW. Visual search and stimulus similarity. Psychological Review. 1989; 96(3): 433-458. [PubMed: 2756067]

Gibson BS, Kingstone A. Visual Attention and the Semantics of Space: Beyond Central and Peripheral Cues. Psychological Science. 2006; 17(7):622-627. [PubMed: 16866749]

Gibson BS, Scheutz M, Davis GJ. Symbolic control of visual attention: Semantic constraints on the spatial distribution of attention. Attention, Perception, \& Psychphysics. 2009; 71(2):363-374.

Griffin IC, Nobre AC. Orienting Attention to Locations in Internal Representations. Journal of Cognitive Neuroscience. 2003; 15(8):1176-1194. [PubMed: 14709235]

Huang L, Pashler H. Working memory and the guidance of visual attention: Consonance-driven orienting. Psychonomic Bulletin \& Review. 2007; 14(1):148-153. [PubMed: 17546745]

Ihssen N, Linden DEJ, Shapiro KL. Improving visual short-term memory by sequencing the stimulus array. Psychonomic Bulletin \& Review. 2010; 17(5):680-686. [PubMed: 21037166] 
Ikkai A, McCollough AW, Vogel EK. Contralateral Delay Activity Provides a Neural Measure of the Number of Representations in Visual Working Memory. Journal of Neurophysiology. 2010; 103:1963-1968. [PubMed: 20147415]

Irwin, DE.; Andrews, RV. Integration and accumulation of information across saccadic eye movements. In: Inui, T.; McClelland, JL., editors. Attention and Performance. Vol. XVI. Cambridge, MA: MIT Press; 1996. p. 125-155.

Jennings JR, Wood CC. The e-adjustment procedure for repeated-measures analyses of variance. Psychophysiology. 1976; 13:277-278. [PubMed: 1273235]

Landman R, Spekreijse H, Lamme VA. The role of figure-ground segregation in change blindness. Psychonomic Bulletin \& Review. 2004; 11(2):254-261. [PubMed: 15260190]

Lee Y, Lee H, Tsai S. Effects of post-cue interval on intentional forgetting. British Journal of Psychology. 2007; 98:257-272. [PubMed: 17456272]

Loftus, GR.; Loftus, EF. Essence of Statistics. 2. New York: Random House; 1988.

Logan GD. Cumulative progress in formal theories of attention. Annual Review of Psychology. 2004; 55:207-234.

Logan GD. Working memory, task switching, and executive control in the task span procedure. Journal of Experimental Psychology: General. 2004; 133:218-236. [PubMed: 15149251]

Logie, RH. Visuo-Spatial Working Memory. Hove, UK: Erlbaum; 1995.

Lovett, MC.; Reder, LM.; Lebiere, C. Modeling Working Memory in a Unified Architecture: An ACTR Perspective. In: Miyake, A.; Shah, P., editors. Models of Working Memory: Mechanisms of Active Maintenance and Executive Control. Newy York, NY: Cambridge University Press; 1999.

Luck SJ. Multiple mechanisms of visual-spatial attention: Recent evidence from human electrophysiology. Behavioural Brain Research. 1995; 71:113-123. [PubMed: 8747179]

Luck SJ. Massachusetts Institute of Technology. An Introduction to the Event-Related Potential Technique. 2005

Luck SJ, Vogel EK. The capacity of visual working memory for features and conjunctions. Nature. 1997; 390:279-281. [PubMed: 9384378]

Makovski T, Jiang YV. Distributing versus focusing attention in visual short-term memory. Psychonomic Bulletin Review. 2007; 14(6):1072-1078. [PubMed: 18229477]

Makovski T, Sussman R, Jiang YV. Orienting attention in visual working memory reduces interference from memory probes. Journal of Experimental Psychology: Learning, Memory, and Cognition. 2008; 34(2):369-380.

Makovski T, Watson LM, Koutstaal W, Jiang YV. Method matters: Systematic effects of testing procedure on visual working memory sensitivity. Journal of Experimental Psychology: Learning, Memory, and Cognition. 2010; 36(6):1466-1479.

Matsukura M, Luck SJ, Vecera SP. Attention effects during visual short-term memory maintenance: Protection or prioritization. Perception and Psychophysics. 2007; 69(8):1422-1434. [PubMed: 18078232]

Miyake A, Friedman NP, Emerson MJ, Witzki AH, Howerter A, Wager TD. The unity and diversity of executive functions and their contributions to complex "frontal lobe" tasks: A latent variable analysis. Cognitive Psychology. 2000; 41:49-100. [PubMed: 10945922]

Miyake, A.; Shah, P. Models of Working Memory. Mechanisms of Active Maintenance and Executive Control. Cambridge University Press; 1999.

Navon D. Resources--a theoretical soup stone? Psychological Review. 1984; 91:216-234.

Nee DE, Jonides J. Dissociable interference-control processes in perception and memory. Psychological Science. 2008; 19(5):490-500. [PubMed: 18466411]

Nunez, PL. Electric Fields of the Brain. New York: Oxford University Press; 1981.

Olivers CNL, Meijer F, Theeuwes J. Feature-Based Memory-Driven Attentional Capture: Visual Working Memory Content Affects Visual Attention. Journal of Experimental Psychology: Human Perception and Performance. 2006; 32(5):1243-1265. [PubMed: 17002535]

Owen A. The Functional Organization of Working Memory Processes Within Human Lateral Frontal Cortex: The Contribution of Functional Neuroimaging. European Journal of Neuroscience. 1997; 9:1329-1339. [PubMed: 9240390] 
Palmer, SE. Vision science: Photons to phenomenology. Cambridge, MA: Bradford Books/MIT Press; 1999.

Proctor RW. Recognition of memory for pictures as a function of poststimulus interval: An empirical clarificaion of existing literature. Journal of Experimental Psychology: Learning, Memory, and Cognition. 1983; 9:256-262.

Schmidt BK, Vogel EK, Woodman GF, Luck SJ. Voluntary and involuntary attentional control of visual working memory. Perception \& Psychophysics. 2002; 64:754-763. [PubMed: 12201334]

Shaffer WO, Shiffrin RM. Rehearsal and Storage of Visual Information. Journal of Experimental Psychology. 1972; 92:292-296. [PubMed: 5058950]

Shaw M. Attending to multiple sources of information: I. The integration of information in decision making. Cognitive Psychology. 1982; 14:353-409.

Sligte IG, Scholte HS, Lamme VA. Are there multiple visual short-term memory stores? PLos ONE. 2008 [Electronic Version].

Sperling G. The information available in brief visual presentations. Psychological Monographs. 1960; 74 (Whole No. 498).

Vogel EK, Machizawa MG. Neural activity predicts individual differences in visual working memory capacity. Nature. 2004; 428(6984):748-751. [PubMed: 15085132]

Vogel EK, McCollough AW, Machizawa MG. Neural measures reveal individual differences in controlling access to working memory. Nature. 2005; 438:500-503. [PubMed: 16306992]

Vogel EK, Woodman GF, Luck SJ. Storage of features, conjunctions, and objects in visual working memory. Journal of Experimental Psychology: Human Perception and Performance. 2001; 27:92114. [PubMed: 11248943]

Vogel EK, Woodman GF, Luck SJ. The time course of consolidation in visual working memory. Journal of Experimental Psychology: Human Perception and Performance. 2006; 32(6):14361451. [PubMed: 17154783]

Washburn DA, Astur RS. Nonverbal working memory of humans and monkeys: Rehearsal in the sketchpad? Memory and Cognition. 1998; 26:277-286. [PubMed: 9584435]

Wilken P, Ma WJ. A detection theory account of change detection. Journal of Vision. 2004; 4(12): 1120-1135. [PubMed: 15669916]

Woodman GF. A brief introduction to the use of event-related potentials in studies of perception and attention. Attention, Perception, \& Psychophysics. 2010; 72(8):2031-2046.

Woodman GF, Luck SJ. Serial deployment of attention during visual search. Journal of Experimental Psychology: Human Perception and Performance. 2003; 29:121-138. [PubMed: 12669752]

Woodman GF, Luck SJ. Do the contents of visual working memory automatically influence attentional selection during visual search? Journal of Experimental Psychology: Human Perception and Performance. 2007; 33(2):363-377. [PubMed: 17469973]

Woodman GF, Vecera SP, Luck SJ. Perceptual organization influences visual working memory. Psychonomic Bulletin \& Review. 2003; 10:80-87. [PubMed: 12747493]

Woodman GF, Vogel EK. Selective storage and maintenance of an object's features in visual working memory. Psychological Bulletin \& Review. 2008; 15(1):223-229.

Woodman GF, Vogel EK, Luck SJ. Visual search remains efficient when visual working memory is full. Psychological Science. 2001; 12:219-224. [PubMed: 11437304]

Zacks, RT.; Hasher, L., editors. Directed ignoring: Inhibitory regulation of working memory. Vol. xiv. San Diego: San Diego, CA, US: Academic Press; 1994.

Zhang W, Luck SJ. Sudden Death and Gradual Decay in Visual Working Memory. Psychological Science. 2009; 20(4):423-428. [PubMed: 19320861] 


\section{A Baseline Trials}
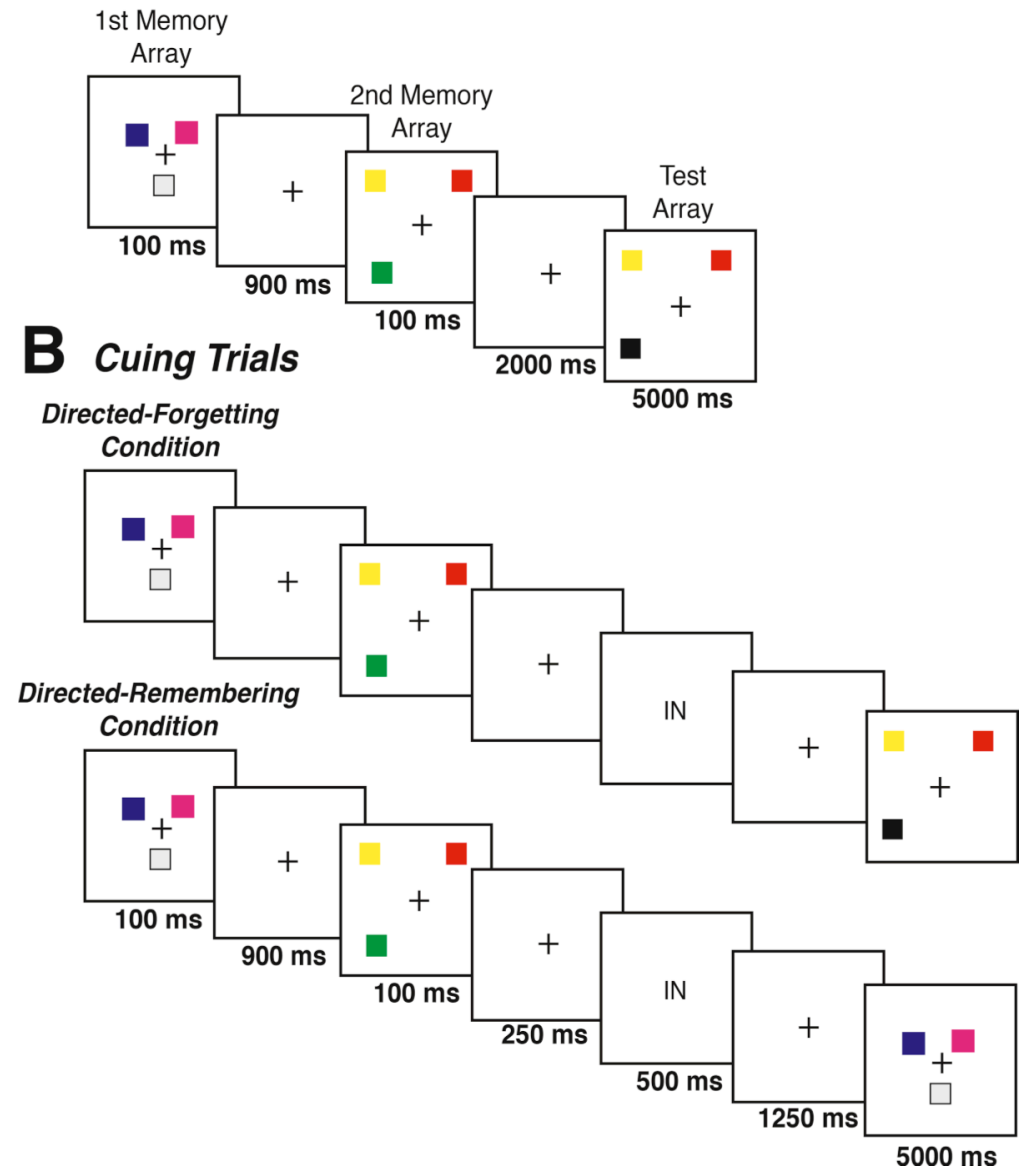

Figure 1.

Example of the sequence of stimuli presented during baseline trials (A) and cuing trials in the Directed-Forgetting and Directed-Remembering Conditions (B) of Experiment 1. 


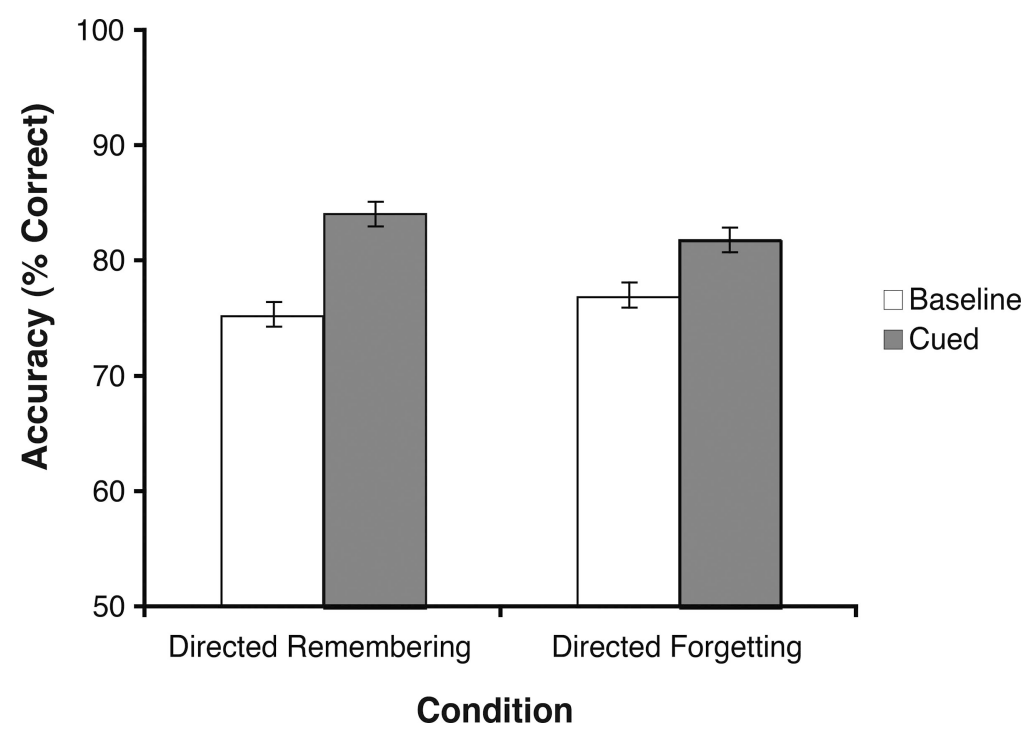

Figure 2.

Mean accuracy (percent correct) as a function of experimental condition and cue presentation. The error bars show the $95 \%$ within subject's confidence intervals in this and subsequent figures (Loftus \& Loftus, 1988). 


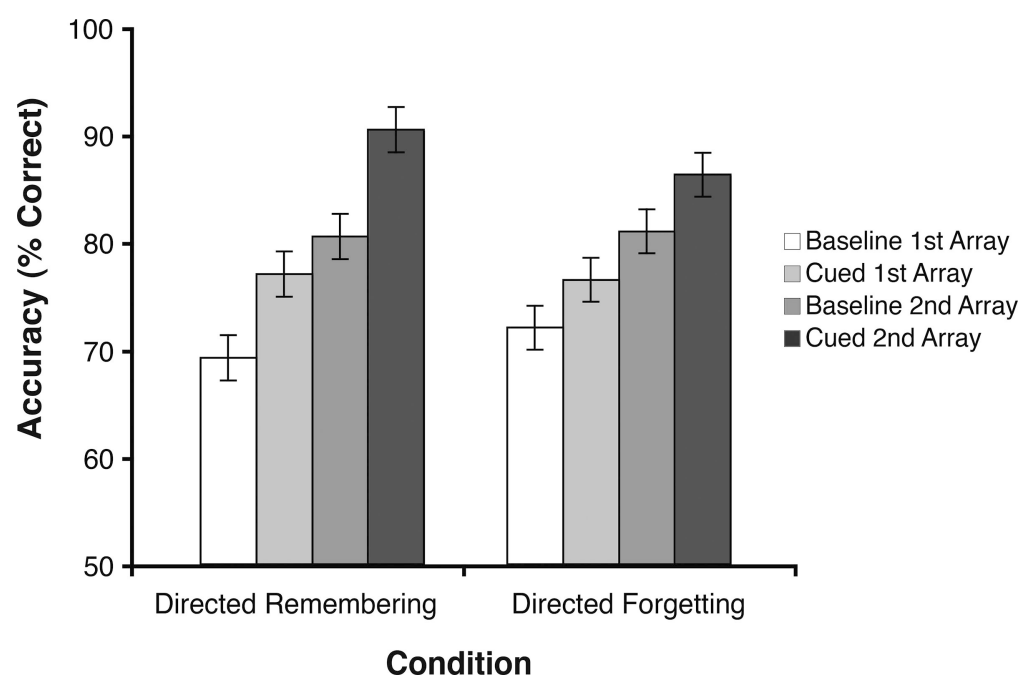

Figure 3.

Mean accuracy (percent correct) as a function of experimental condition, cue presentation, and array presentation order. The left two bars in each condition represent performance when the first array was tested. The right two bars in each condition represent performance when the second array was tested. 


\section{A Baseline Trials}
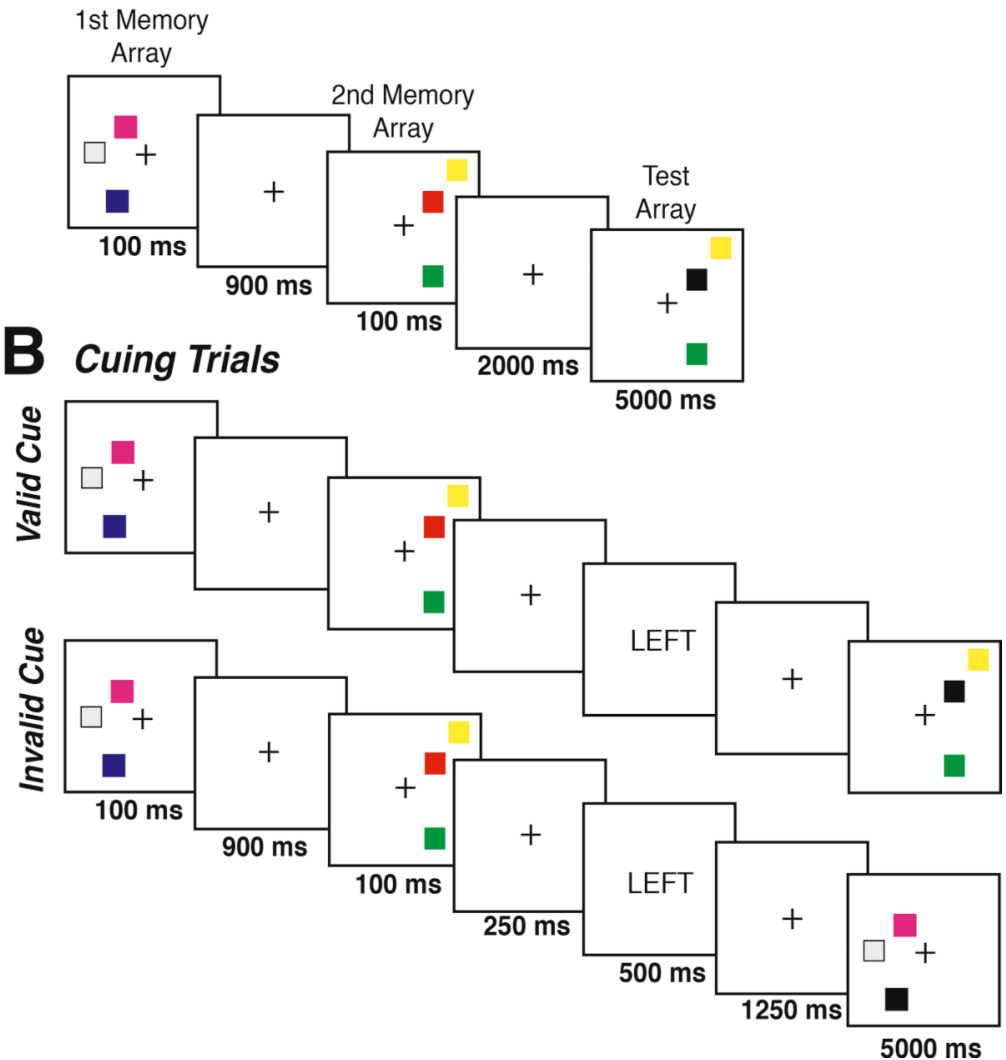

Figure 4.

Example of the sequence of stimuli presented during baseline trials (A) and trials with valid and invalid directed-forgetting cues (B) of Experiment 2. 


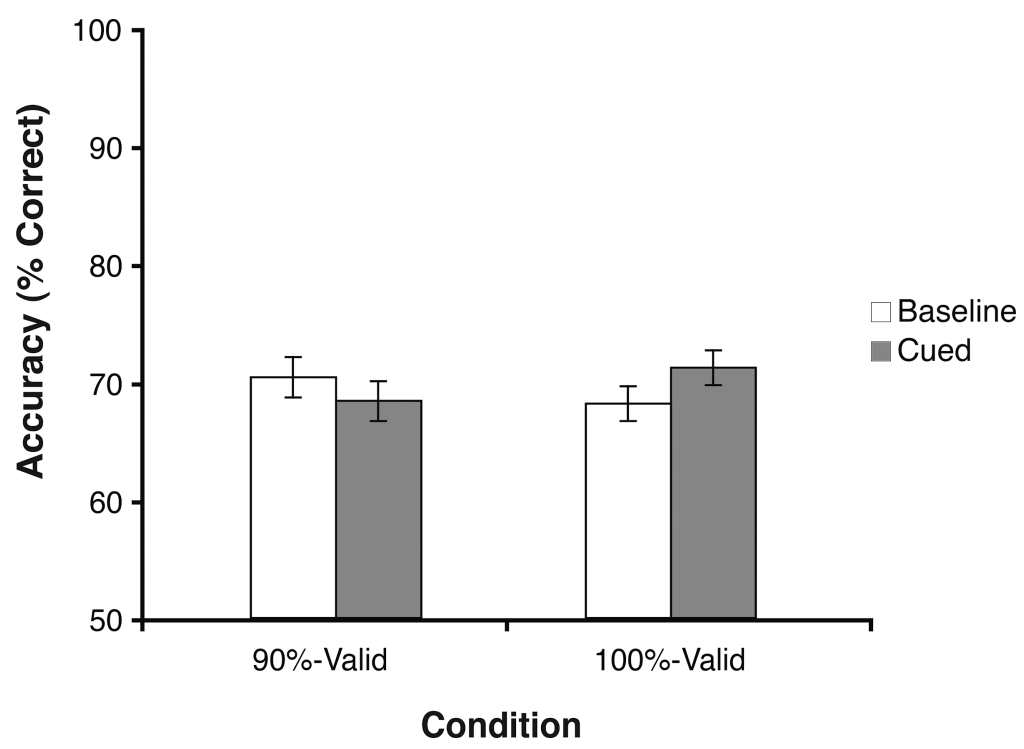

Figure 5.

Mean accuracy (percent correct) as a function of experimental condition and cue presentation. 
A

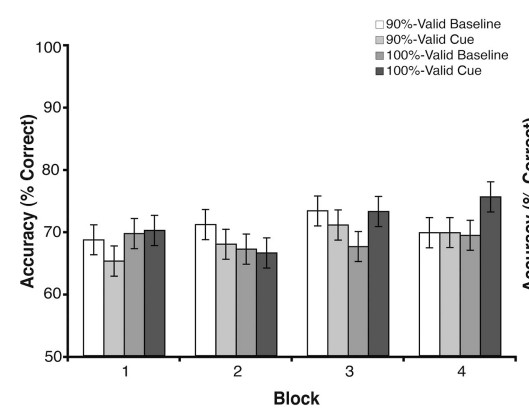

B

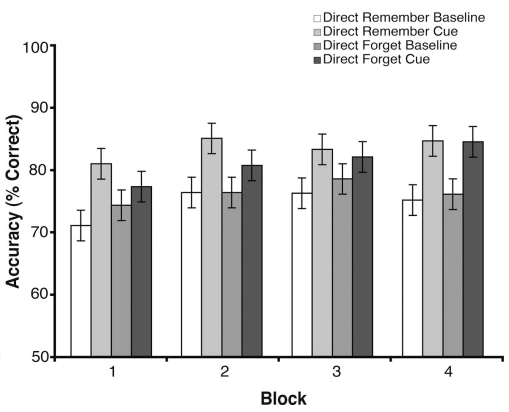

Figure 6.

Mean accuracy as a function of experimental condition, cue presentation, and trial block accuracy. (A) The block-by-block analysis from Experiment 2. (B) The block-by-block analysis from Experiment 1. 


\section{Baseline Trials}

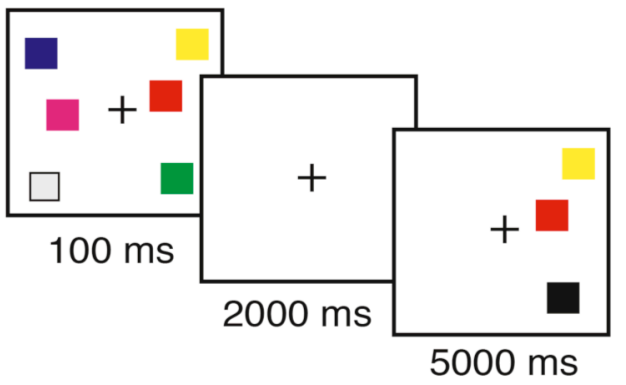

Cued Trials

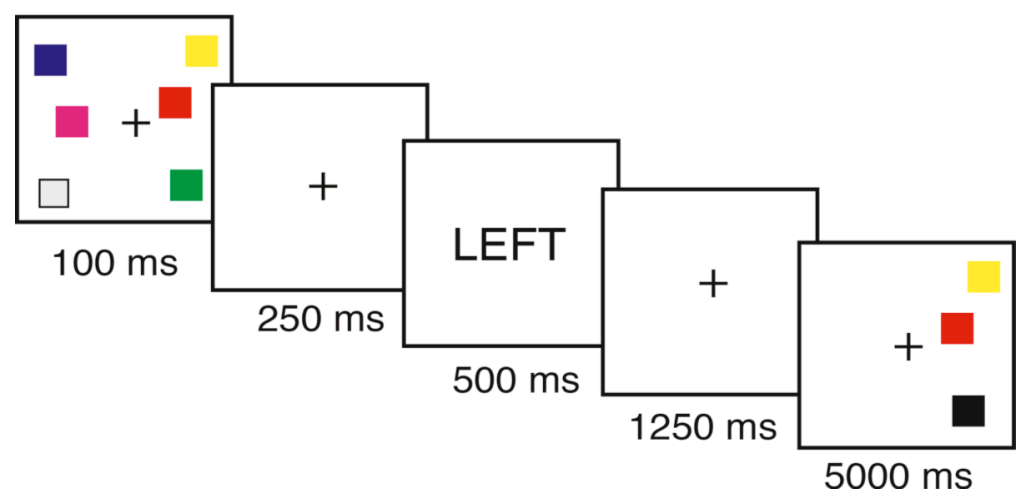

Figure 7.

Example of the sequence of stimuli presented during Experiment 3. The cued trials of the directed-remembering condition would show the word "right" for this stimulus sequence. 
A Directed-Forgetting
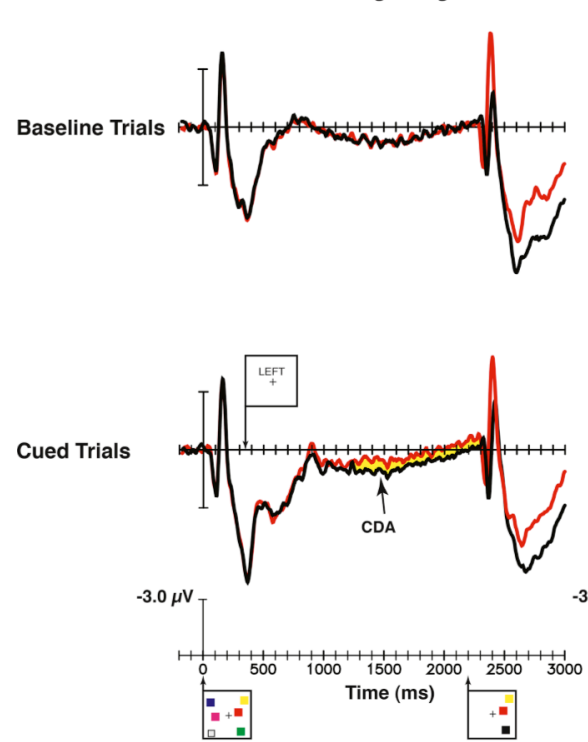

B Directed-Remembering
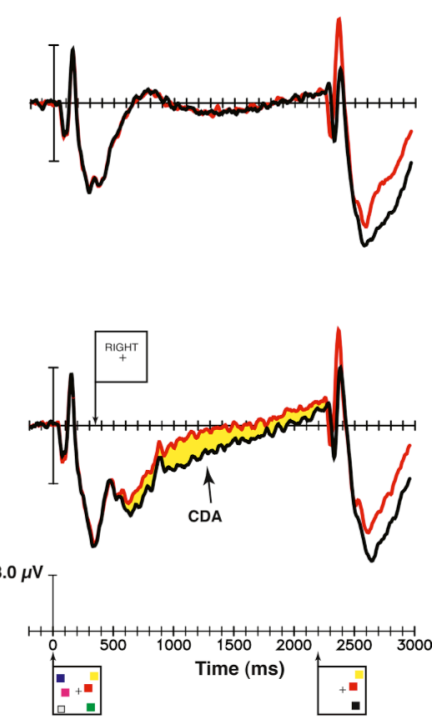

Contralateral to Tested Items

Figure 8.

The ERP results of Experiment 4 time locked to memory array onset. (A) The waveforms from electrodes $\mathrm{T} 5 / 6$ relative to the tested items on uncued-baseline trials and trials with directed-forgetting cues. (B) The waveforms from T5/6 relative to the tested items on uncued-baseline trials and trials with directed-remembering cues. The yellow region shows the measurement window used to quantify the amplitude of the CDA. 


\section{Table 1}

Mean accuracy (in percent correct \pm SEM) across the trials in which the first versus second array was tested in the baseline, no-cue trials and trials with directed forgetting cues in Experiment 2.

\begin{tabular}{|c|c|c|c|c|}
\hline & \multicolumn{2}{|c|}{ First Array } & \multicolumn{2}{c|}{ Second Array } \\
\hline Experimental Condition & Baseline & Cued & Baseline & Cued \\
\hline $90 \%$ Invalid & $66.7 \pm 1.7 \%$ & $65.4 \pm 2.1 \%$ & $74.2 \pm 1.8 \%$ & $72.6 \pm 2.5 \%$ \\
\hline $100 \%$ Invalid & $64.3 \pm 1.7 \%$ & $67.4 \pm 2.1 \%$ & $72.4 \pm 15 \%$ & $75.5 \pm 2.6 \%$ \\
\hline
\end{tabular}




\section{Table 2}

Mean contralateral delay activity onset latency (in ms) for cued trials by condition for Experiment 3 .

\begin{tabular}{|c|c|c|}
\hline & \multicolumn{2}{|c|}{ Latency } \\
\hline Experimental Condition & Mean & SEM \\
\hline Directed Forgetting & 1701.8 & 84.8 \\
\hline Directed Remembering & 1378.9 & 88.5 \\
\hline
\end{tabular}

